\title{
A Test Architecture for V-2-X Cooperative Systems Field Operational Tests
}

\author{
Andrea Tomatis*, Markus Miche ${ }^{\dagger}$, Florian Haeusler ${ }^{\ddagger}$, \\ Massimiliano Lenardi*, Thomas Michael Bohnert ${ }^{\dagger}$, and Ilja Radusch ${ }^{\ddagger}$ \\ * Hitachi Europe, 955, Routes des Lucioles, F-06560 Valbonne - Sophia Antipolis, France \\ Email: \{andrea.tomatis, massimiliano.lenardi\}@ hitachi-eu.com \\ $\dagger$ SAP Research CEC Zurich, Kreuzplatz 20, 8008 Zurich, Switzerland \\ Email: \{markus.miche, thomas.michael.bohnert\}@sap.com \\ $\ddagger$ Fraunhofer Institute, Kaiserin-Augusta-Allee 31, 10589 Berlin, Germany \\ Email: \{florian.haeusler, ilja.radusch\}@fokus.fraunhofer.de
}

\begin{abstract}
In recent years, several research projects have discovered the potential of Intelligent Transport Systems (ITS) and have developed corresponding demonstrators for Vehicle-toVehicle (V2V) and Vehicle-to-Infrastructure (V2I) communication, which act as proof of concepts.

This paper provides the required test infrastructure to go beyond a pure proof of concept by enabling the validation of a complete V2X communication system that covers V2V and V2I communications plus the interconnection of vehicles and backend systems known as Vehicle-to-Business (V2B) communication. We present an integrated test architecture that allows not only the evaluation of vehicular communications on test tracks but moreover paves the way for conducting real life Field Operational Tests (FOT) with thousands of vehicles based on state-of-the-art broadband wireless access technologies. In addition, this paper presents the selection procedure applied for the identification of hardware and software components required for realizing the V2X system based on a predefined set of Use Cases (UCs).
\end{abstract}

\section{INTRODUCTION}

In the context of European R\&D projects related to vehicular cooperative systems, PRE-DRIVE C2X project [1] is paving the road towards future Field Operational Tests (FOTs) covering Vehicle-to-Vehicle (V2V), Vehicle-to-Infrastructure (V2I), and Vehicle-to-Business (V2B) communication [2]. PRE-DRIVE C2X is a European project funded by the European Commission Information Society and Media among the 7th Framework initiatives. It is coordinated by Daimler AG and its consortium includes OEMs, technology and automotive suppliers, research institutes, and universities. The mission of this project is to verify and validate the extended European Intelligent Transport System (ITS) architecture defined within the COMeSafety project [3] hence providing evidence of its conceptual and technological maturity. For that purpose, a set of Use Cases (UCs) representative for the overall scope of $\mathrm{V} 2 \mathrm{X}$ communication is analyzed, prototypically realized, and evaluated [4].

This paper presents the selection procedure applied for the identification of hardware and software components required for realizing the V2X system satisfying the selected UCs. Moreover, it defines the test architecture, which aims at providing an infrastructure to evaluate and validate a complete ITS system, in particular the PRE-DRIVE C2X system

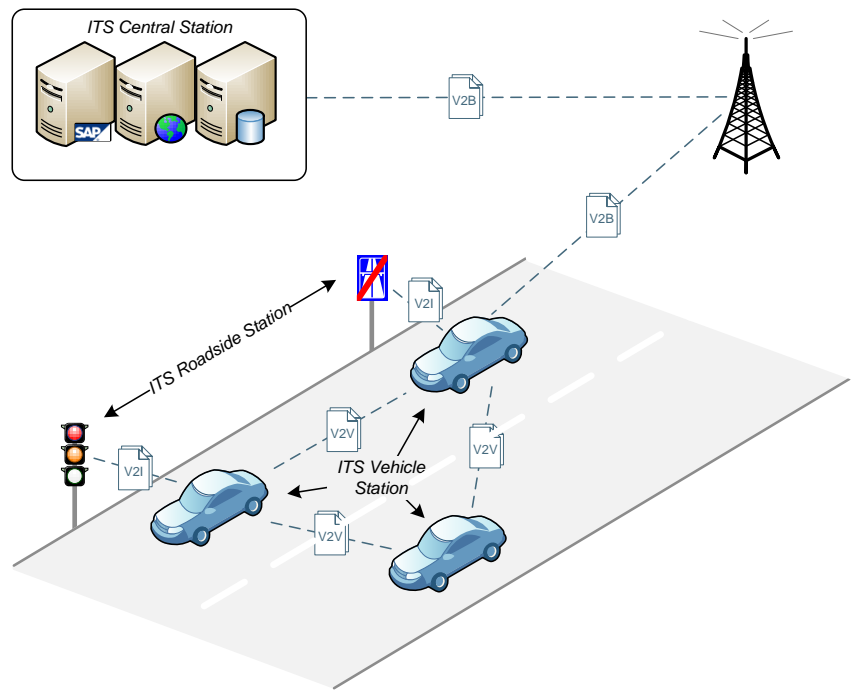

Fig. 1. A complete Intelligent Transportation System consisting of V2V, V2I, and V2B Concepts

described in [5]. This test architecture does not only include technical tests, but also traffic efficiency and safety impact analysis, and user acceptance tests.

There are various methodological approaches available for testing IT systems, such as Model-based testing [6], testdriven development [7], or the V-Model [8]. Based on the latter model, PRE-DRIVE C2X aims at integrating and testing of the ITS architecture. Since the system under test ranges from a complete ITS architecture with multiple, distributed ITS stations, to singular ITS stations and software components, standard test tools satisfy only individual needs. We designed an integrative test system, which supports efficient management, development, execution, and analysis of tests. It is specifically tailored to the broad range of required tests to validate all aspects of a V2X-based ITS architecture.

\section{OVERVIEW OF ITS ARCHITECTURE}

To provide a pan-European ITS architecture that enables the different vehicular communications outlined in Figure 1, 


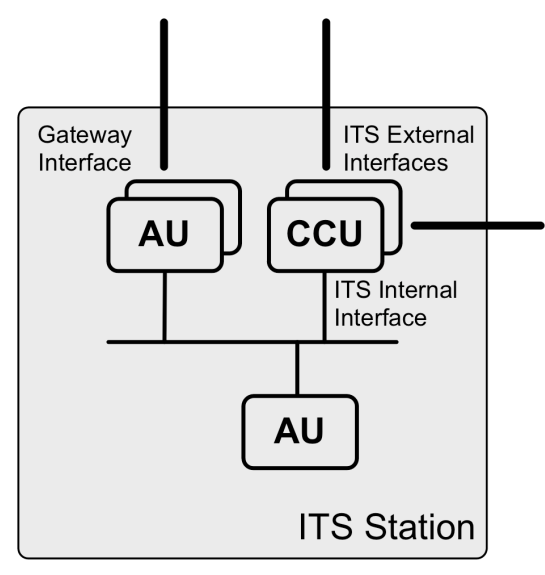

Fig. 2. ITS station

PRE-DRIVE C2X refines and complements the common European ITS communication architecture defined within the COMeSafety project [3]. This system architecture [5] specifies the concepts of cooperative systems and shows the reference protocol architecture for an ITS station. The ITS station is a generic component for vehicles and roadside communication infrastructure, which consists of Communication \& Control Units (CCU) and Application Units (AU) as shown in Figure 2.

Together they form a network inside the ITS station where CCUs obtain connectivity to the ITS network via one or more egress interfaces. Different instantiations of the ITS station exist (as shown in 1):

- ITS Vehicle Station is mounted within or fixed to a vehicle and enables the vehicle to participate in cooperative ITS applications.

- ITS Roadside Station is a fixed installation along the road which to participate in cooperative ITS applications.

- ITS Personal Station is a consumer electronics device, which is typically assigned to a person, e.g. pedestrian or cyclist. In case the consumer electronics device is linked to a vehicle, it is considered as ITS vehicle station ${ }^{1}$.

- ITS Central Station covers diverse back-end system reaching from traffic management systems to business systems such as for instance electronic toll collect systems or insurance applications for realizing novel usagebased insurance concepts [9].

According to different scenarios, the ITS architecture can be modified and adapted to specific economical and regulatory conditions. Each ITS station refers to a protocol stack which follows the ISO/OSI reference model, vertically extended by a management layer and a security layer. The ITS protocol stack is based on routing and packet transport protocols specifically designed for wireless multi-hop communication utilizing geographical positions [2]. This approach allows high mobility of vehicles, scalability with the number of network nodes, and meets application requirements in particular for road safety.

\footnotetext{
${ }^{1}$ In the paper the ITS personal station will not be considered for the selection procedures and for the description of the ITS test architecture
}

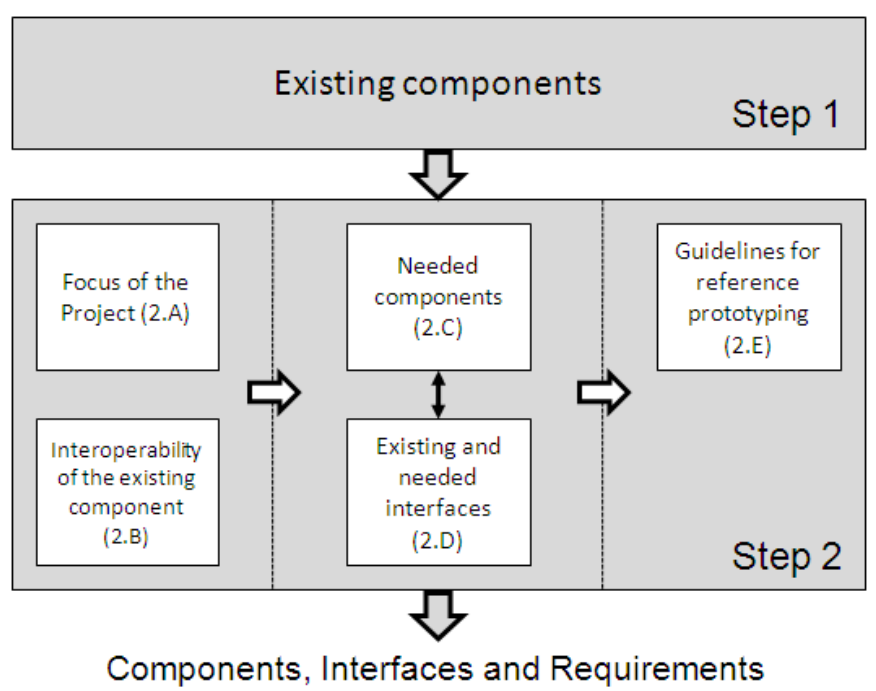

Fig. 3. Two step selection procedure

IP packet transport is assured either by means of encapsulation and tunneling over the ad hoc network for $\mathrm{V} 2 \mathrm{~V}$ and V2I communication, or by using the public access network. Moreover, it includes design concepts for, e.g., data congestion control, interworking between ad hoc and infrastructure-based communication, interworking between different wireless technologies, and others. In this protocol architecture, the security is guarantee with the adoption of security procedures, e.g. cryptography, privacy, and identity management.

V2B communication covers the interconnection of vehicle and back-end systems and constitutes an extension of the European ITS communication architecture defined in [3]. It provides the foundation for a outstanding data exchange between vehicles and back-end systems and paves the way for novel business models as well as improvements of existing business processes. Vehicle Relationship Management [10], usage-based insurance concepts such as Pay As You Drive [9], and City Traffic Control service provide three examples that demonstrate the overall potential of $\mathrm{V} 2 \mathrm{~B}$ communication for both service providers and drivers as the actual end customers.

$\mathrm{V} 2 \mathrm{~B}$ requires the protocol stack outlined above to be extended by dedicated communication facilities such as an asynchronous messaging support, an appropriate discovery mechanism, and a priority-based message queue. That way, the intermittent connectivity of vehicles is taken into account and an agile and scalable interconnection between vehicles and back-end systems is enabled.

\section{A. Selection procedure of main architectural components}

In order to implement an ITS system able to cover diverse UCs, the selection of required hardware and software components has been conducted according to a dedicated two steps procedure shown in Figure 3.

In the first step, we analyzed other ITS projects with respect to existing components related to the PRE-DRIVE $\mathrm{C} 2 \mathrm{X}$ architecture. For each component we identified the main 
functionalities as well as the respective external interfaces.

Within the second step, which is the core of the selection procedure, a subset of components, based on the survey performed in step 1, were chosen for realizing the architecture. Since the interconnection of vehicles and back-end systems represents a novel aspect that has not been dealt with in other projects so far, also new components have been specified. The second step of the selection procedure is divided in the following five tasks:

Focus of the project (2.A). Based on the results of step 1, this task provides a list of essential components needed to develop the PRE-DRIVE C2X architecture taking into account the selected use cases.

Interoperability of the existing component (2.B). This task reviews the status of existing ITS prototypes and their potential with respect to their re-use in our project. In addition, the task examines available prototypes for selected use cases on a case by case basis. Specific emphasis is put on components that are available as subsystem, but have not yet been integrated in the existing prototypes.

Needed components (2.C). According to step 2.A, this task specifies the needed components in detail.

Existing and needed interfaces (2.D). Considering the essential components selected by step 2.A, this task deals with the identification of interfaces. Therefore, interfaces of different components are analyzed and adapted to the PREDRIVE C2X scope. Finally, the task provides the needed interfaces and specifies them in collaboration with the step 2.C.

Guidelines for reference prototyping (2.E). Taking into account the selected components, this task defines the requirements to assure that all system components are robust enough to sustain a field trial under real life conditions.

A first overview of the existing components shows that most of the components seem already available from other projects. However, those components cannot be considered interoperable. In fact, due to the correlations among components of the same available demonstrator, the re-use of such components is not possible. Moreover, results show that from a hardware perspective, a significant part of the use case needs are covered by the existing $\mathrm{C} 2 \mathrm{C}-\mathrm{CC}$ prototypes used during the $\mathrm{C} 2 \mathrm{C}-\mathrm{CC}$ Demo 2008 [11]. This is confirmed by investigating re-use potential from both an architectural, as well as from a use case point of view. On the contrary, from a software perspective many simplifications are in place in the existing prototypes and not each needed functionality is already implemented (e.g. the communication facilities required for V2B communication).

Finally, concerning the interfaces analysis, the results show that components related to network interface cards, antenna, and hardware platforms provide well-defined interfaces (antenna, data bus, etc.) of various types. On the other hand, network interface cards provide standardized software interfaces (e.g., IEEE 802.2 LLC for 802-type of devices), but these interfaces need to be extended for their usage for road safety applications. Regarding sensor components (e.g., vehicle's CAN bus) well-defined interfaces are available. However, prototype implementations of sensors currently use other interfaces, such as serial RS-232 or Ethernet interfaces.

Separate attention needs the interaction between the different layers of the protocol architecture. In fact, data transport, network, security, as well as facility-related components use proprietary, non-standardized interfaces, which makes the interoperability of components difficult.

Finally, interfaces for the test system or for the interconnection of vehicle and back-end systems constitute a novel aspect that has not been covered in detail by European research projects on cooperative systems so far.

\section{ITS TEST ARCHITECTURE}

Based on the validation objectives of PRE-DRIVE C2X, we designed three non-congruent test environments. These are the simulation environment, the test bench environment, as well as the FOT environment. Each environment has its own test purpose, strengths, weaknesses, and limitations. In the following description, we will focus on the FOT environment but give a short overview on the complete test infrastructure, its use and interplay.

Simulation environment. Multiple simulators (traffic, network, environment, and application simulator) are coupled to provide a fully virtual environment. There are two main objectives: First, it should pre-evaluate certain aspects of the implementation (e.g. correct interface implementations) in order to limit the tests in the FOT environment. Second, the simulation is suited to run tests, which could not even be evaluated during the FOT, e.g. traffic efficiency effects with thousands of vehicles.

Test bench environment. The test bench environment provides the possibility to validate the system under test under laboratory conditions. The main objective is to ensure that singular ITS stations including their sub-components work correctly, especially all external interfaces of ITS vehicle and roadside stations. The basic architecture and data flows are described in Figure 4. The white boxes belong to the ITS system in use. Grey and dark grey boxes are functional blocks of the test bench environment. Each ITS station is connected to a test unit - that is the On-board Testing Unit (OBTU) for the ITS vehicle station, the Roadside Testing Unit (RSTU) for the ITS roadside station, and the ITS Central Station Testing Unit (CSTU) for the ITS central station, respectively. These testing units are software components deployed directly on the ITS stations in PRE-DRIVE C2X. They provide the communication link to the test bench and are the interface to the ITS station. This link is needed to transfer log data and monitoring data to the test bench for test run analysis and validation. The difference between log data and monitoring data is, that monitoring data is transferred in real time for test run observation. Log data includes the complete data, which is recorded during a test run. Thus, monitoring data is a subset of log data. Continuous lines illustrate common data flows of the operational system, dashed or dotted lines show the testspecific data flows in the test bench environment. The test bench includes three components. The Virtual Data Injector is a component, which provides all kind of data to the ITS 


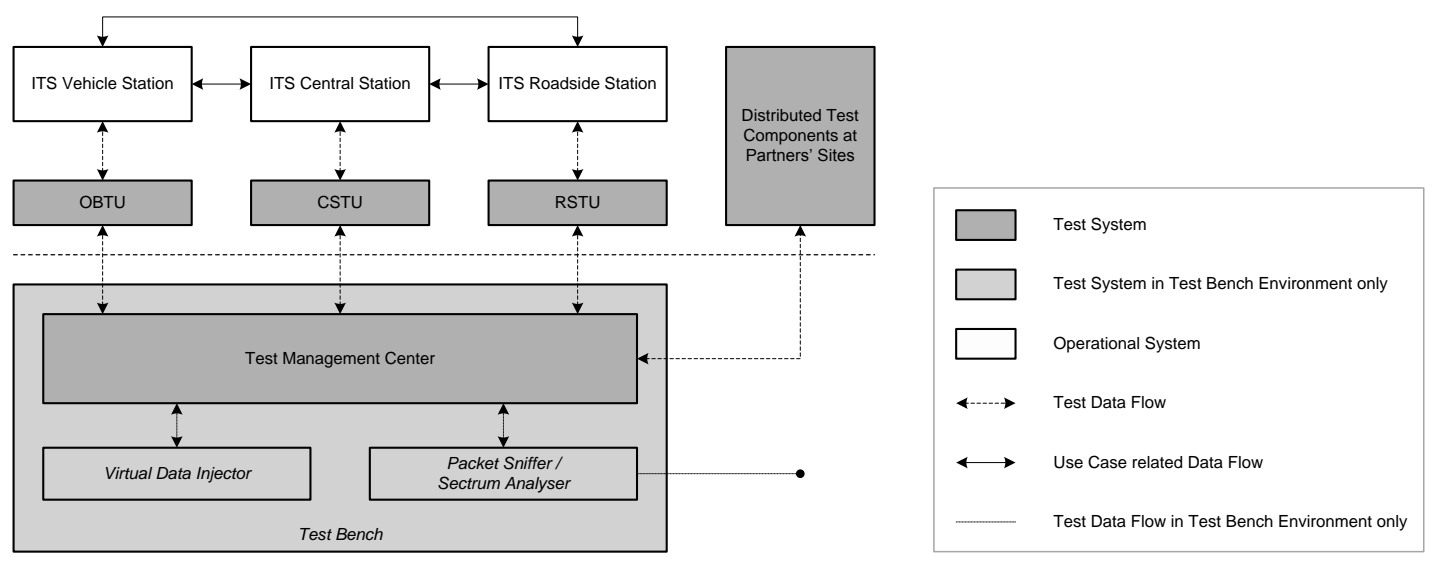

Fig. 4. Test Bench and FOT Environment

station, which is not available under laboratory conditions (e.g. GPS data or data from the vehicles' CAN bus). This data has been recorded in advance and is used here to put the system under test into a real-world state.

FOT environment. This environment is suited to run test with moving vehicles and human vehicle drivers on closed test tracks and public roads. It is suited to evaluate safety, (limited) traffic efficiency impact, and business system based on the ITS. All communication aspects, driver interactions, and vehicle dynamics could be considered and evaluated. The FOT test system includes a central point of control and observation, the Test Management Center (TMC). Moreover, it consists of the testing units for ITS vehicle and roadside stations outlined afore (OBTU, RSTU, CSTU).

The combination of these test environments provides an extensive test system for evaluating and validating V2X communications and applications enabled by them. Employing multiple environments has the advantage, that different aspects of component or system tests can be addressed efficiently. In order to realize a test system, which is neither over-engineered nor turns out to be to light weighted, we specified the test system based on representative test purpose descriptions and accordingly derived requirements for the relevant test environment. An in-depth description will be provided in [12].

\section{A. FOT Test Management Center}

The TMC is the central point of test control and observation. It is employed in the test bench as well as in the FOT environment. We will introduce it in the context of the FOT environment.

The TMC is the only test specific central component. It interacts with ITS stations as outlined in Figure 4 not considering the test bench specific components in light grey. The TMC is required to support testers in controlling and configuring relevant ITS stations, monitoring tests, developing and editing test cases, save and deploy software for ITS stations. Furthermore, the TMC serves as a data sunk for live monitoring and $\log$ data to OBTUs, RSTUs, and CSTUs. Figure 5 shows the internal structure and the components of the TMC including information on provided and accessed interfaces.

The Test Data Exchange component receives, initiates the storage, and parses live monitoring or log files plus received from ITS stations. Moreover, it provides identity management functionality and is able to process register requests sent by ITS stations. The Test Data Exchange component further sends the control data to relevant ITS stations. It proves two interfaces to the Test Data Database and to the Test Monitoring component.

The Test Data Database provides the functionality to store, retrieve, delete, and update all test specific data. It provides an interface, which is used by the Test Control System, and an external interface, especially to retrieve data for detailed test evaluation.

The Test Monitoring component monitors and visualizes test runs. Therefore, it receives live monitoring data (such as the current position of test vehicles) via the Test Data Exchange component. Additionally, it has test specification data, that is data determining how the test run should be executed. Thus, the component has information about target and actual states. It serves two purposes: The component monitors the deviation between actual and target states and triggers the Test Control System to intervene, in case the deviation exceeds a previously defined tolerance. In addition to that, the component visualizes the actual and the target states to support testers in deciding whether the test is running as planned or whether they have to intervene the test run.

The Test Control System controls the execution of the test. That means it initiates actions to intervene and influence test runs. Deviations between target and actual states detected by the monitoring component (or the monitoring tester), the Test Control Component deduces actions (or takes them from testers), and sends them to relevant testing unit. Examples for actions are instructions to test drivers to go faster or slower.

The Deployment System manages software components deployed at the RSTU, OBTU or CSTU. It supports software version management and the automatic deployment of software to multiple testing units. 


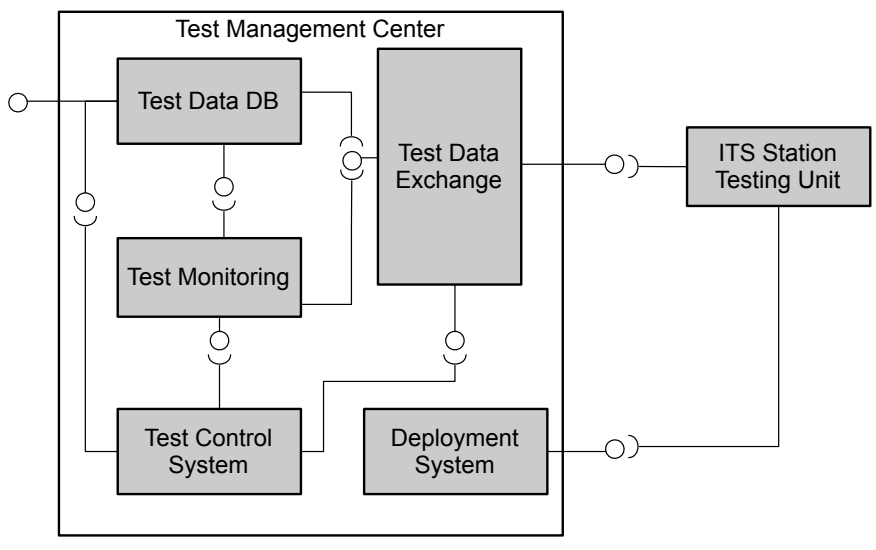

Fig. 5. Test Management Center

\section{B. ITS Station Testing Unit}

The Station Testing Unit (i.e., RSTU, OBTU, or CSTU) is the point of access to the ITS station for the TMC. This component logs information and controls the test execution. In general, the testing unit is an application running on top of the ITS protocol stack (as any PRE-DRIVE C2X application). The computational resources as well as the Human Machine Interface (HMI), e.g. half screen for test and half screen for the UC, are shared. Both shared and dedicated communication channel could be used to transfer real time monitoring data from the TMC and receive commands from the TMC. In addition a dedicated storage is requested since a huge quantity of data has to be stored.

Figure 6 shows the main components of the testing unit, how the components are connected between each other (inter-unit interface) and how the testing unit is interfaced with the ITS station (intra-unit interface) and the TMC (external interface).

The Test Control is in charge of the overall control of the testing unit. It is able to manage different tasks for guaranteeing a correct test execution. In addition, it manages commands sent by the TMC, it facilitates software updates and it provides Application Programming Interfaces (APIs).

The Communication Component makes use of dedicated communication facilities such as an asynchronous messaging support, an appropriate discovery mechanism, and a prioritybased message queue to manage the communication channel with the TMC. More details on this component can be found in Section III-C.

The HMI Component provides a bidirectional, programming language independent information exchange between vehicle applications and in-vehicle HMIs. In particular it decouples the application logic from any OEM-specific HMI properties and moreover allows OEMs for optimizing the user interface with respect to their display capabilities and input modalities.

The Feeding and Controlling Component provides the necessary functionalities to feed the ITS station with virtual data. Moreover, it is able to provide APIs to control and manage the Test Case execution.

The Logging and Parsing Component provides logging

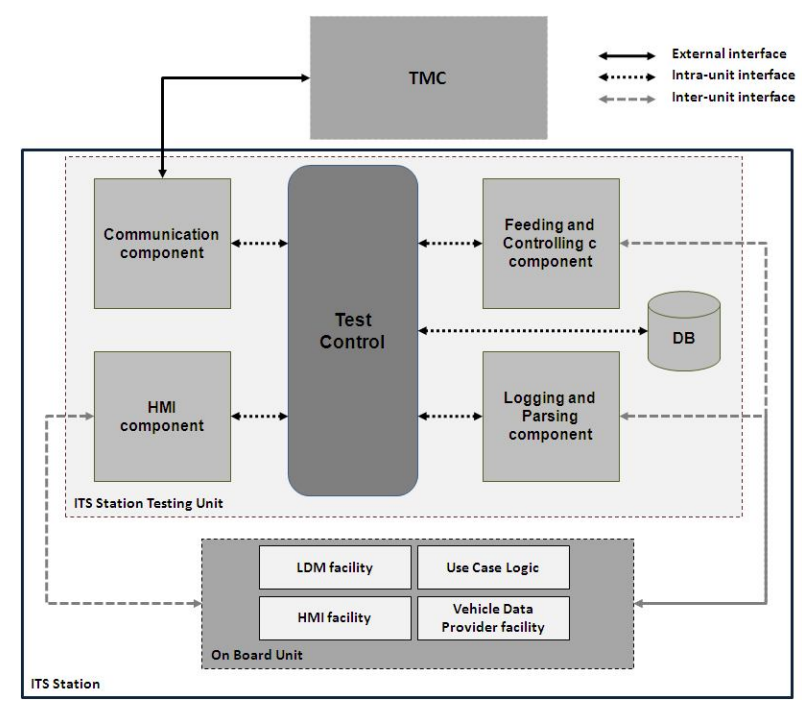

Fig. 6. Testing Unit on the ITS station

and parsing capabilities to collect data from the facility and application layers.

The $D B$ Component provides the functionalities for datamanipulation. Specifically, it interacts with the database to store, retrieve, update, dump, and backup data.

\section{Communication Component}

In the FOT environment, several vehicles that are driving either on test tracks or public roads will be tested and monitored by the TMC. The related information exchange between vehicles and the TMC will be based on state-of-theart Broadband Wireless Access (BWA) technologies such as for instance UMTS.

However, the intermittent connectivity of vehicles caused by their high mobility and the incomplete network coverage of existing BWA technologies constitute a central challenge of the FOT. The non-permanent connectivity of vehicles further affects the reliability of the actual message transmission. To enable and ensure an end-to-end Quality of Service, specific in-vehicle functionality for priority-based queuing and rescheduling of message delivery is required. Finally, since in PRE-DRIVE C2X the message exchange between vehicles and the TMC will not be based on appropriate communications protocols such as Mobile IP but on standard IPv4, a respective discovery mechanism is needed.

For that reason, the FOT requires a dedicated in-vehicle communication component that copes with the challenges outlined above. The building blocks of this module are depicted in Figure 7. Indeed, this component covers a subset of the facilities realized by the communication component Back-end Integration Manager specified in [5], which is used for the interconnection of vehicles and back-end systems (for more information, see [10]).

The vehicle event handler of the communication component of the ITS station testing unit realizes an asynchronous, eventbased message exchange between vehicles and the TMC 


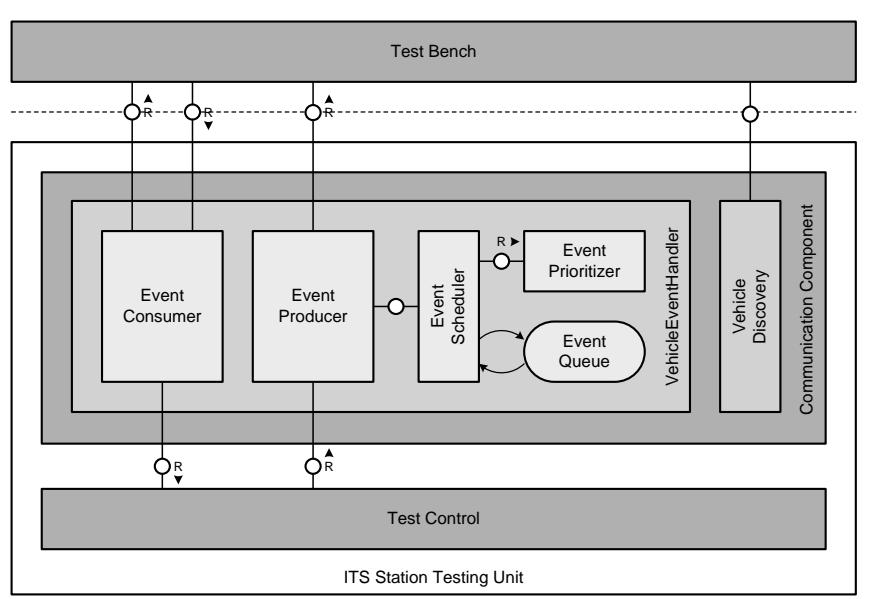

Fig. 7. Communication Component of the ITS Station Testing Unit

according to the interaction style publish/ subscribe. The different classes of test data (e.g., virtual data or monitoring data) are reflected by corresponding topics. Moreover, the vehicle event handler comprises a priority-based message queue plus a scheduler, which manages the message delivery pursuant to the assigned priorities. Thus, the communication component accounts for the varying priorities of test data that are exchanged between vehicles and the TMC during the FOT.

As stated afore, the information exchange in the FOT is based on IPv4 within the scope of PRE-DRIVE C2X. The respective requirements are covered by the vehicle discovery component, which ensures that in case of an interrupted Internet connection of a vehicle, the new IP address of the latter is registered/ updated in the Test Data Exchange component of the TMC as soon as the connection is reestablished (see also III-A).

That way, the communication component of the ITS station testing unit provides dedicated mechanisms to cope with the specific challenges of the FOT. As an example, all messages a vehicle not connected to the TMC (e.g., because it is driving in a tunnel) tries to send are queued and prioritized by the vehicle event handler. As soon as the vehicle is able to reestablish an Internet connection, it registers itself with the Test Data Exchange component of the TMC. Thereafter, all messages are delivered to the TMC according to their priorities and the vehicle is able to receive new test instructions.

\section{CONCLUSION}

The paper describes the procedure applied within the PREDRIVE C2X project to select the components for the implementation of the ITS architecture, the test environments, and the tools to validate it.

The results of the selection procedure show that some components are already available from other past or ongoing ITS projects. However, most interfaces of these components have to be either adapted or defined from scratch. In particular, the interaction between the different layers of the protocol stack need to be revised.
For the ITS test system, the V-model concept is applied to the ITS world. According to the validation objectives of PRE-DRIVE C2X, the presented approach includes three non-congruent test environments: the simulation environment, the test bench environment, and the FOT environment. The additional value is the applicability of the ITS test system to a real life FOT (moving vehicles plus human vehicle drivers on closed test tracks and public roads). This facilitates the evaluation of the correctness as well as the impact of the ITS architecture on safety, traffic efficiency, and business services (i.e., its business potential).

Looking into the future, PRE-DRIVE C2X will proceed deploying the described ITS architecture step by step. First, the complete ITS protocol stack will be developed. Then a reference ITS station (prototype) will be realized and validated on top of a specified hardware platform. In parallel, the ITS test system will be implemented and validated. Finally, after the definition of concrete test cases, the prototype will be validated in the simulation and test bench environment as well as using the depicted FOT test system. It is planned to present an analysis of these results in a future paper.

\section{ACKNOWLEDGMENT}

The authors wish to thank the European Commission Information Society and Media for founding PRE-DRIVE C2X Project and the partners who contributed to the deliverables D3.1 and D4.2.

\section{REFERENCES}

[1] "PRE-DRIVE C2X Project - Preparation for Driving implementation and Evaluation of Car-2-X communication technology." [Online]. Available: www.pre-drive-c2x.eu

[2] "ETSI TC ITS - Intelligent Transport Systems." [Online]. Available: www.etsi.org/WebSite/Technologies/IntelligentTransportSystems.aspx

[3] "COMeSAFETY Project, European ITS Communication Architecture Version 2.0." [Online]. Available: www.comesafety.org

[4] "PRE-DRIVE C2X Deliverable D4.1: Detailed Description of Selected Use Cases and Corresponding Technical Requirements," 2008. [Online]. Available: www.pre-drive-c2x.eu

[5] "PRE-DRIVE C2X Deliverable D1.2: Refined Architecture," 2008. [Online]. Available: www.pre-drive-c2x.eu

[6] A. Pretschner and J. Philipps, "Methodological issues in model-based testing," pp. 281-291, 2004.

[7] Beck, "Test driven development: By example," Boston, MA, USA, 2002.

[8] "The V-Modell XT," 2009. [Online]. Available: http://v-modell.iabg.de/ v-modell-xt-html-english/index.html

[9] M. Ott, H. Gretzinger, T. Ippisch, T. Dalcher, V. Bättig, and A. Bereuter, "Erhöhte sehschärfe - technologiebasierte innovationen in der versicherungswirtschaft: Bedeutung, chancen und herausforderungen," Accenture Studie, 2008.

[10] M. Miche and T. M. Bohnert, "The internet of vehicles or the second generation of telematic services," ERCIM News, vol. 77, pp. 43-45, 2009.

[11] ETSI, "ETSI TR 102698 V1.1.1 - Intelligent Transport Systems (ITS); Vehicular Communications; C2C-CC Demonstrator 2008; Use Cases and Technical Specifications ," ETSI TC ITS, Tech. Rep., 2008. [Online]. Available: webapp.etsi.org/workprogram/Report_WorkItem. asp?WKI_ID=30939

[12] "PRE-DRIVE C2X Deliverable D4.2: Requirements and specification of testing architecture and procedures and requirements and specification of test management centre," 2009. [Online]. Available: www.pre-drive-c2x. $\mathrm{eu}$ 